\title{
BISTUA
}

\section{RMR y tipo de explosivo: incidencia en las voladuras subterráneas}

\author{
RMR and type of explosive: incidence in underground blasting
}

\author{
Álvaro Correa Arroyave ${ }^{a}$; Jorge Eric Rueda Fonseca ${ }^{b}$ \\ ${ }^{a}$ Ingeniero de Minas y Metalurgia, Doctor-Ingeniero en Mecánica de Rocas, Especialista en Técnicas de voladura a cielo abierto y subterráneo, \\ Docente Universidad Santo Tomás, Bogotá; Docente Escuela de Ingenieros Militares, Bogotá, Colombia, \\ ${ }^{b}$ Geólogo, Especialista en Técnicas de voladura en obras de ingeniería civil y militar, Escuela de Ingenieros Militares, Bogotá, Colombia
}

Correspondencia: alvarocorreaa@usantotomas.edu.co ; jorge.eric.rueda.fonseca@gmail.com

Recibido: Enero 16, 2021. Aceptado: Febrero 10, 2021. Publicado: Junio 19, 2021

\section{Resumen}

La clasificación de masas rocosas de Bieniawski, RMR -Rock Mass Classifications, junto con la Q de Barton y el GSI de Hoek, los tres sistemas de clasificación más frecuentemente utilizados en muchos países incluyendo a Colombia como ayuda para determinar un sinnúmero de propiedades del macizo rocoso, que de otra forma serían muy tediosas y costosas de determinar. Estas características permiten prever los tiempos de autosoporte de una excavación subterránea, así como su tipo de sostenimiento, velocidad de avance y complejidad de construcción y con base en ellos determinar costos y tiempos de excavación bastante aproximados de estas estructuras, y aunque se recomienda utilizar el método de avance de perforación y voladura para macizos rocosos con RMR de I, II y III, en la bibliografía no se encuentra ninguna relación directa entre el RMR y las dimensiones del burden, del espaciamiento ni del retaque. También es cierto que la mayoría de las expresiones propuestas para determinar estos parámetros (burden, espaciamiento y retaque) solo contemplan la densidad de la roca y del explosivo; algunas pocas, la velocidad de las ondas en el medio y otras más, la resistencia a la compresión o a la tracción. Por otro lado, las excavaciones subterráneas se caracterizan por su estado de confinamiento debido a la profundidad, por la forma y tamaño del área a excavar, así como por parámetros geométricos inherentes al proceso de perforación tales como el diámetro, la longitud, la dirección y la ausencia de una cara libre. Estas condiciones insinúan que, en los diseños de la malla de perforación y voladura subterránea, debería reflejarse la influencia de estos parámetros. Los resultados de la investigación que venimos realizando nos permite proponer una expresión que conlleva las propiedades dinámicas a tracción del macizo rocoso para estar acorde con las características dinámicas generadas por el explosivo, el cual también ingresa como una variable importante; además, estamos considerando el nivel de acoplamiento de las cargas, las dimensiones de la sección del túnel, sus condiciones de confinamiento y el RMR en el que se encuentra inmerso.

Palabras clave: RMR, Burden, Indugel Plus AP, ANFO, Resistencia a tracción dinámica de la roca intacta y del macizo rocoso.

\section{Abstract}

Bieniawski's rock mass classification, RMR -Rock Mass Classification- is, together with Barton's Q and Hoek's GSI, the three most frequently used classification systems in many countries including Colombia as an aid to determine several rock mass properties, which would otherwise be very tedious and costly to determine. These characteristics allow predicting the selfsupporting times of an underground excavation, as well as its type of support, speed of advance and complexity of construction and based on them determine costs and excavation times of these structures as accurately as possible, and although it is recommended to use the drilling and blasting advance method for rock massifs with RMR of I, II and III, in the literature no direct relationship is found between the RMR and the dimensions of the burden, spacing or the stemming. It is also true that most of the expressions proposed to determine these parameters (burden, spacing, and stemming) only consider the density of the rock and the explosive; a few, the velocity of the waves in the medium and others, the compressive or tensile strength. On the other hand, underground excavations are characterized by their confined state due to depth, by the shape and size of the area to be excavated, as well as by geometrical parameters inherent to the drilling process such as diameter, length, direction, and the absence of a free face. These conditions imply that the influence of these parameters should be reflected in the designs of the underground drilling and blasting mesh. The results of the research we have been carrying out allow us to propose an expression that considers the dynamic tensile properties of the rock mass to be in accordance with the dynamic characteristics generated by the explosive, which also enters as an important variable; in addition, we are considering the level of explosive load coupling, the dimensions of the tunnel section, its confinement conditions and the RMR in which it is immersed.

Keywords: RMR, Burden, Indugel Plus AP, ANFO, Dynamic tensile strength of intact rock and rock mass. 


\section{Introducción}

En la Ref.[1] propusimos una expresión para el cálculo del burden y demás parámetros de diseño de una voladura subterránea, después de una búsqueda bastante detallada que nos llevó a considerar una decena de expresiones recomendadas para la determinación de dichos parámetros; en el presente, no obstante, solo haremos referencia a las expresiones más utilizadas[2], Konya Ec.(1), Ash Ec.(2), Langefors Ec.(3) y López Jimeno Ec.(4), las cuales se trascriben en su orden, las cuales se trascriben en su orden:

donde:

$$
B=\left(\frac{2 \rho_{e}}{\rho_{r}}+1,5\right) \times d
$$

B: Burden

$\rho_{e}$ : Densidad del explosivo

$\rho_{r}$ : Densidad de la roca

D: Diámetro de la carga (pulg)

$$
B=0,1584 \times D_{e x p} \times\left(\frac{\rho_{e} V_{d}^{2}}{\rho_{r}}\right)^{1 / 3}
$$

donde:

\section{B: Burden}

$\mathrm{D}_{\text {exp: }}$ : Diámetro del explosivo

$\rho_{e}$ : Densidad del explosivo

$\rho_{r}$ : Densidad de la roca

$\mathrm{V}_{\mathrm{d}}$ : Velocidad de detonación

$$
B_{\max }=\frac{D}{33} \sqrt{\frac{\rho_{e} \times P R P}{\bar{c} \times f \times(S / B)}}
$$

donde:

$\mathrm{B}_{\text {max }}$ : Burden Máxima (m)

D: Diámetro del barreno (mm)

$\bar{C}$ : Constante de la roca

f: Factor de fijación

S/B: Relación entre espaciamiento/burden

$\rho_{e}$ : Densidad de carga $\left(\mathrm{kg} / \mathrm{dm}^{3}\right)$

PRP: Potencia relativa en peso del explosivo

donde:

$$
B=0,88 \times q_{f}^{0,35}, q_{f}=7,85 \times 10^{-4} \times d^{2} \times \rho_{e}
$$

\section{B: Burden}

$q_{f}$ : Carga de Fondo

d: Diámetro de explosivo

$\rho_{e}$ : Densidad del explosivo

Una mención especial haremos de la expresión propuesta por Pearse, para la cual Borquez propuso una metodología muy interesante a fin de determinar el $K_{v}$, en lugar de utilizar los valores propuestos por Pearse [3] y ratifica la necesidad de determinar la resistencia a la tracción dinámica de la roca. En buena medida, esta expresión sirvió de punto de partida para nuestra investigación, considerando parámetros dinámicos no de la roca intacta sino del macizo rocoso, tal como lo ilustraremos más adelante, a más de otros parámetros asociados con la sección y la profundidad del túnel.

$$
B=K_{v} \times 10^{-3} \times D \times\left(\frac{P D}{R T}\right)^{\frac{1}{2}}
$$

La expresión que propusimos en su momento [1] es:

$$
B=\frac{K_{A} \times K_{S R F} \times K_{R M R b} \times D}{1000} \sqrt{\frac{P D}{R_{t d m}}}
$$

En el artículo en mención se definen los parámetros de esta expresión y se presentan los valores propuestos para las constantes $\mathrm{K}_{\mathrm{A}}, \mathrm{K}_{\mathrm{SRF}}$ y $\mathrm{K}_{\mathrm{RMRb}}$. El avance en nuestra investigación, particularmente en lo referente a las propiedades dinámicas del macizo rocoso y las condiciones de desacoplamiento de las cargas, así como la validación de los valores de las constantes, anteriormente propuestas, nos ha permitido proponer la nueva relación, ecuación (14).

\section{Planteamiento del problema}

Para el diseño de una voladura subterránea, se encuentra en la bibliografía técnica un sinnúmero de expresiones que permiten determinar el burden, y a partir de él, los demás parámetros de la voladura tales como el espaciamiento y el retaque con ayuda de algunas tablas anexas; no obstante, la mayoría de ellas no hacen intervenir los parámetros más relevantes tanto de la roca intacta como del macizo rocoso y menos aún de sus comportamientos dinámicos; tampoco conocemos de ninguna expresión que haga intervenir parámetros asociados con los niveles de confinamiento del macizo rocoso. Estas falencias conducen, a nuestro criterio, a voladuras ineficientes y a que se escuchen, aún en escenarios medianamente técnicos, reglas de dedo que parecieran responder de igual manera para todos los macizos rocosos no importando sus propiedades genéticas, litológicas o su grado de fracturamiento natural.

La bibliografía técnica, lo advierte [4] los parámetros de la roca y del macizo rocoso que intervienen en una buena perforación y voladura son, para la perforación: dureza, resistencias, elasticidad, plasticidad, abrasividad, textura y estructura, y para la voladura: [2] densidades, resistencias dinámicas, porosidad, fricción interna, conductividad, composición y explosiones secundarias de polvo. Por su parte, las propiedades de los macizos rocosos que mayor incidencia tienen son: litología, fisuras preexistentes, presencia de agua, esfuerzos in-situ y temperatura [2].

Las expresiones que gobiernan los diseños de voladura subterránea deben considerar no solo las características de los explosivos y de la roca intacta, sino también las condiciones reales del macizo rocoso y el factor de acoplamiento de las cargas, lo cual hará que dicho diseño sea más eficiente y eficaz, reduciendo los consumos específicos y minimizando los impactos negativos de las voladuras.

La propuesta que hacemos en este artículo es una respuesta a esa necesidad sentida, en el entendido que hoy en día la práctica de la perforación y la voladura pasó de ser una práctica rudimentaria sin muchos fundamentos técnicos, a un 
arte soportado en el conocimiento científico de todas las variables que inciden en un eficiente diseño del proceso desde la perforación, hasta los resultados de una buena voladura.

\section{Objetivo}

Proponer una expresión que permita calcular el burden, y mediante él, otros parámetros de una voladura subterránea tales como el espaciamiento y el retaque de todos los barrenos exceptuando los de precorte, los cuales tienen un tratamiento especial del que nos ocuparemos en una próxima oportunidad. De su puesta en práctica, se podrán esperar los siguientes resultados:

- Reducción del número de perforaciones

- Reducción del consumo de explosivos

- Reducción del tiempo dedicado a las operaciones de perforación y voladura

- Mayor eficiencia en el proceso de perforación y voladura

- $\quad$ Reducción de la sobre excavación

- Reducción de los efectos nocivos generados por las vibraciones

\section{Metodología}

Este trabajo de investigación documental se soporta en una metodología de búsqueda en la bibliografía técnica, la cual permitió la identificación de cuatro importantes variables: las características de la roca intacta, las del macizo rocoso, las del explosivo y los parámetros del túnel relacionados con su sección y profundidad. El plan de trabajo, entonces, se compone de dos etapas, la primera corresponde a la caracterización de los materiales, roca, macizo rocoso y explosivo, así como a las características del túnel; una vez se halla la expresión que los relaciona, la segunda corresponde a la convalidación de sus resultados obtenidos al confrontarlos con expresiones, gráficas y tablas, así como con patrones de voladura citados en la bibliografía técnica.

\section{Desarrollo de la expresión}

\subsection{Resistencia a la tracción dinámica de la roca intacta}

Cuando se trata de analizar los efectos producidos por la voladura, conviene determinar las características dinámicas de las rocas, particularmente a tracción, puesto que el explosivo genera ese mismo tipo de esfuerzos, dinámicos mas no, estáticos. Escudriñando en la bibliografía encontramos que el profesor Carlos López Jimeno en Manual de Perforación y Voladura de Rocas enuncia al respecto..." El tratamiento racional de los problemas reales obliga a considerar las resistencias dinámicas, ya que éstas aumentan con el índice de carga, pudiendo llegar a alcanzar valores entre 5 y 13 veces superiores a las estáticas" [2].

Debe aclararse que los parámetros dinámicos de la roca determinados en laboratorio no son representativos por la heterogeneidad y anisotropía de los macizos rocosos y por la imposibilidad de reproducir los ensayos en condiciones similares a los períodos de carga de una voladura que son inferiores a 0,1 ms. En ocasiones, los valores de laboratorio han superado a los obtenidos "in situ" en proporciones de 5 a 8 , siendo las macrofisuras y los esfuerzos residuales en el macizo rocoso, los responsables de tales discrepancias.

\subsubsection{Rotura y resistencia de la roca}

Cuando una roca recibe un estado tensional proporcionado por una detonación, tiende a romperse por cizallamiento; el confinamiento hace que ese cizallamiento sea más difícil (aumentando su fricción y resistencia). En voladuras subterráneas ese confinamiento es todavía más acentuado por lo que es importante tenerlo en cuenta cuando se diseña la construcción de un túnel. Otro factor de influencia en la resistencia es el tiempo de aplicación de la carga. La resistencia aumenta cuanto menor es el tiempo del ciclo de carga, lo que sucede con la onda de choque producida por un explosivo.

Una onda de choque plana cuando entra en contacto con la roca, le aplica una compresión que la deforma de una manera idéntica a un ensayo de compresión con confinamiento lateral. Para que haya fragmentación de la roca, sin deformación elástica, la presión tiene que ser suficientemente alta $\approx 2 *$ (resistencia con confinamiento).

Como se indicó antes, las propiedades de la roca intacta que influyen en los procesos de perforación y voladura incluyen muy particularmente la densidad, la velocidad de las ondas en ese medio y la resistencia a la compresión y a la tracción, pero muy particularmente la resistencia a la tracción dinámica, propiedad esta última que es bastante difícil de determinar, por lo que se recurrió a una búsqueda bibliográfica, en la que encontramos la siguiente expresión citada por Borquez [3]:

$$
\sigma_{t d r i}=4,5 \sigma_{\text {teri }}
$$

Otra expresión, propuesta por Yoandro Diéguez-García et al. [5] determina el límite de resistencia a la tracción dinámica ante cargas explosivas mediante un índice de dinamicidad que modifica la resistencia a la tracción estática de la roca intacta y que está definido con base en la velocidad sónica y el peso unitario de la misma, mediante la relación:

$$
\begin{gathered}
{\left[\sigma_{t d r i}\right]=K_{t d r i}\left[\sigma_{\text {teri }}\right],} \\
\text { donde, } \quad k_{t d r i}=3.44-0.2 \times 10^{-10} \rho_{r} V_{L}^{2}
\end{gathered}
$$

Nos acogimos a esta expresión tanto para la determinación de la resistencia a tracción dinámica de la roca intacta, como para la del macizo rocoso, tal como lo indicaremos posteriormente.

5.2. Resistencia a la tracción dinámica del macizo rocoso 
Tal como se ha venido insistiendo en este escrito, las propiedades que deben tenerse en cuenta cuando se trata de enfrentar un problema de perforación y voladura de rocas, compete a las propiedades del macizo rocoso y no a las de la roca intacta, es por ello por lo que igualmente nos dimos a la tarea de investigar sobre la forma de determinar la resistencia a la tracción estática del macizo rocoso, a partir de la resistencia a la tracción estática de la roca intacta. Después de una exhaustiva búsqueda dimos con las siguientes expresiones que nos permitieron resolver dicha incógnita [6]:

$$
\begin{gathered}
\sigma_{\text {temr }}=\sigma_{\text {teri }} \times e^{\left(\frac{R M R-100}{27}\right)} \\
\sigma_{t m}=\frac{\sigma_{c i}}{2}\left(m_{b}-\sqrt{m_{b}^{2}+4 s}\right) \quad ; \quad \sigma_{t}=-\frac{S \sigma_{c i}}{m_{b}}
\end{gathered}
$$

Para nuestros cálculos, tomamos la primera de estas expresiones que está más directamente relacionada con el RMR, objeto de nuestro estudio.

Ahora, para hallar $\sigma_{t d m r}$ utilizamos la constante $k_{t d m r}$ a partir de la $k_{t d r i}$ al considerar la Tabla 1 en la que las propiedades del macizo rocoso en su densidad y velocidad longitudinal se modificaron en función del valor de su RMR. De esta forma encontramos el valor de la resistencia a la tracción dinámica del macizo rocoso, mediante la expresión:

$$
\left[\sigma_{t d m r}\right]=k_{t d m r}\left[\sigma_{t e m r}\right]
$$

\begin{tabular}{|c|c|c|c|c|c|}
\hline \multicolumn{2}{|c|}{ RMR } & Reducción de la $\rho_{\mathrm{T}}$ & pr $\mathrm{Kg} / \mathrm{m}^{3}$ & Reducción de la V1 & $\mathrm{V} / \mathrm{m} / \mathrm{s}$ \\
\hline \multicolumn{4}{|c|}{ Granito $\rho_{\mathrm{r}}: 2650 \mathrm{~kg} / \mathrm{m} 3$} & \multicolumn{2}{|l|}{ Vl: $5250 \mathrm{~m} / \mathrm{s}$} \\
\hline Ia & $91-100$ & $5 \%$ & 2518 & $5 \%$ & 4988 \\
\hline $\mathrm{Ib}$ & $81-90$ & $10 \%$ & 2385 & $10 \%$ & 4725 \\
\hline Па & $71-80$ & $15 \%$ & 2253 & $15 \%$ & 4463 \\
\hline IIb & $61-70$ & $20 \%$ & 2120 & $20 \%$ & 4200 \\
\hline IIIa & $51-60$ & $25 \%$ & 1988 & $25 \%$ & 3938 \\
\hline $\mathrm{II} \mathrm{b}$ & $41-50$ & $30 \%$ & 1855 & $30 \%$ & 3675 \\
\hline
\end{tabular}

Tabla 1. Modificación de la densidad y velocidad longitudinal del macizo rocoso en función de su RMR. Caso de un granito.

Peso unitario: $2650 \mathrm{~kg} / \mathrm{m}^{3}$ Velocidad sónica: $5250 \mathrm{~m} / \mathrm{s}$

En la Tabla 2 ilustramos algunas propiedades físicomecánicas de algunos tipos litológicos de rocas, citadas por González de Vallejo [7], los cuales pueden servir de guía para conocer los parámetros de una voladura eficiente.

\subsection{Caracterización del explosivo}

El tipo de explosivo y cargue de los barrenos, es el tercer parámetro de importancia en este estudio pues de su Poder de Detonación, función de su densidad y velocidad de detonación, así como de su nivel de acoplamiento, dependerá su poder rompedor. Al respecto, consultamos la Guía del Explosivista de INDUMIL, 2009, [8], Tabla 3 y el Manual para explosivos industriales, INDUMIL, 2018 [9], Tabla 4 a partir de las cuales tomamos las propiedades de algunos explosivos para realizar un ejercicio que servirá para convalidar los resultados de la propuesta que estamos haciendo en el presente estudio.

Tabla 2. Propiedades físico-mecánicas medias de algunos tipos de rocas.

\begin{tabular}{|c|c|c|c|c|c|c|c|c|c|}
\hline \multicolumn{7}{|c|}{ Propiedades físico-mecánicas de algunos tipos de rocas } \\
\hline Litología & I & II & III & IV & V & VI & VII & VII & IX \\
\hline Arenisca & 2450 & 97,5 & 13 & 2800 & 39,78 & 13 & 3,06 & 3,11 & 40 \\
\hline Basalto & 2800 & 140 & 15 & 5500 & 26,25 & 3 & 1,75 & 2,73 & 8 \\
\hline Caliza & 2450 & 100 & 17 & 4250 & 43,35 & 17 & 2,55 & 2,68 & 46 \\
\hline Cuarcita & 2650 & 260 & 20 & 5750 & 33,8 & 20 & 1,69 & 1,94 & 39 \\
\hline Diabasa & 2900 & 295 & 55 & 6250 & 64,35 & 55 & 1,17 & 1,5 & 83 \\
\hline Dolomía & 2550 & 130 & 15 & 5500 & 28,5 & 15 & 1,9 & 2,12 & 32 \\
\hline Gabro & 3050 & 245 & 22 & 5500 & 34,98 & 22 & 1,59 & 1,86 & 41 \\
\hline Gneiss & 2850 & 130 & 13 & 4300 & 31,07 & 13 & 2,39 & 2,54 & 33 \\
\hline Granito & 2650 & 135 & 16 & 5250 & 31,68 & 9 & 1,98 & 2,19 & 20 \\
\hline Mármol & 2700 & 160 & 13 & 4750 & 28,86 & 13 & 2,22 & 2,4 & 31 \\
\hline Lutita & 2400 & 30 & 6 & 2200 & 19,26 & 6 & 3,21 & 3,24 & 19 \\
\hline Pizarra & 2600 & 95 & 14 & 4250 & 35 & 14 & 2,5 & 2,63 & 37 \\
\hline Yeso & 2300 & 25 & 2 & 3500 & 5,76 & 2 & 2,88 & 2,96 & 6 \\
\hline
\end{tabular}

I. Densidad, $\rho_{\mathrm{r}}, \mathrm{Kg} / \mathrm{m}^{3}$

II. Resistencia a compresión simple, $\sigma_{\text {ceri }}, \mathrm{Mpa}$

III. Resistencia a la tracción, $\sigma_{\text {teri, }}, \mathrm{Mpa}$

IV. Velocidad de propagación, $\mathrm{Vl}, \mathrm{m} / \mathrm{s}$

V. Resistencia a la tracción dinámica de la roca intacta $\sigma_{\text {tdri, }}, \mathrm{MPa}$

VI. Resistencia a la tracción estática del macizo rocoso $\sigma_{\text {temr }}, \mathrm{Mpa}$

VII. Constante de transformación roca intacta, $\mathrm{k}_{\mathrm{drri}}, \mathrm{kg} / \mathrm{m}^{*} \mathrm{~s}^{2}$

VIII. Constante de transformación para el macizo rocoso* $\mathrm{k}_{\mathrm{dtmr}}, \mathrm{kg} / \mathrm{m}^{*} \mathrm{~s}^{2}$

IX Resistencia a la tracción dinámica del macizo rocoso $\sigma_{\mathrm{tdmr}}, \mathrm{Mpa}$

Tabla 3. INDUMIL Características de algunos explosivos, 2009

\begin{tabular}{|c|c|c|c|c|c|c|c|}
\hline \multicolumn{8}{|c|}{ Tipo de explosivo } \\
\hline \multicolumn{2}{|c|}{ ANFO } & \multicolumn{2}{|c|}{ Indugel Plus AP } & \multicolumn{2}{|c|}{ Emulind-E } & \multicolumn{2}{|c|}{ Emulind-B } \\
\hline $\begin{array}{l}\text { Densidad, } \\
\text { ol/ }\end{array}$ & Velocidad & Densidad, & Velocidad & Densidad, & Velocidad & Densidad, & $\begin{array}{l}\text { Velocidad } \\
\end{array}$ \\
\hline$\frac{\mathrm{g} / \mathrm{cm}^{3}}{0,05 \pm 0,05}$ & $\frac{\mathrm{m} / \mathrm{s}}{3000 \pm 300}$ & $\frac{\mathrm{g} / \mathrm{cm} \mathrm{m}^{3}}{1,20 \pm 0,003}$ & $\frac{\mathrm{m} / \mathrm{s}}{4500 \pm 500}$ & $\frac{\mathrm{g} / \mathrm{cm}^{3}}{1,16 \pm 0,06}$ & $\frac{\mathrm{m} / \mathrm{s}}{4600 \pm 600}$ & $\frac{\mathrm{g} / \mathrm{cm}^{3}}{1,15 \pm 0.05}$ & $\begin{array}{c}\frac{\boldsymbol{m} / \mathrm{s}}{5300 \pm 300} \\
\end{array}$ \\
\hline
\end{tabular}

\begin{tabular}{|c|c|c|c|c|c|}
\hline \multicolumn{5}{|c|}{ Tipo de explosivo } \\
\hline \multicolumn{2}{|c|}{ ANFO } & \multicolumn{2}{c|}{ Indugel Plus AP } & \multicolumn{2}{c|}{ Emulind-e } \\
\hline Densidad, g/cm & Velocidad $\mathrm{m} / \mathrm{s}$ & Densidad, $\mathrm{g} / \mathrm{cm}^{3}$ & Velocidad $\mathrm{m} / \mathrm{s}$ & Densidad, g/cm & Velocidad $\mathrm{m} / \mathrm{s}$ \\
\hline $0,85 \pm 0,05$ & & $1,21 \pm 0,06$ & $4500 \pm 500$ & $1,19 \pm 0,05$ & $5000 \pm 1000$ \\
\hline
\end{tabular}

Tabla 4. INDUMIL Características de algunos explosivos, 2018

Además, debe tenerse en cuenta el acoplamiento de las cargas, lo que da lugar a encontrar la presión de barreno en función de la presión de detonación y los respectivos diámetros tanto del barreno como del explosivo, lo que lleva a aconsejar un menor diámetro anular para incrementar la presión de barreno. Las expresiones que permiten tener en cuenta estos parámetros son:

$$
\begin{gathered}
P D=0.25 \times \rho_{e} \times V l^{2} \times 10^{-3} \\
P b=P D \times\left(\frac{\emptyset_{e}}{\emptyset_{b}}\right)^{3}
\end{gathered}
$$


Para incrementar la presión de barreno donde esto sea necesario, se acostumbra a combinar explosivos con diferente energía de tensión, por ejemplo, para el cuele o para los pateros. Igual, cuando la columna explosiva está compuesta por una carga de fondo y una carga de columna, se deberán tener en cuenta sus porcentajes a fin de determinar la presión de barreno equivalente; dichos porcentajes afectarán los respectivos valores de densidad y velocidad de detonación. En otros casos, para la línea de voladura adyacente a la de contorno, se emplea, en general, la voladura amortiguada (nosotros la recomendamos).

\subsection{Expresión propuesta}

Después de determinar los valores para las propiedades que ingresarían a la expresión buscada, y teniendo en mente que atrás se indicó que habíamos partido de la expresión de Pearse, concluimos que la expresión propuesta es de la forma:

Siendo,

$$
B=\frac{K_{A} \times K_{S R F} \times K_{R M R b} \times D}{1000} \sqrt{\frac{P b}{\sigma_{t d m r}}}
$$

$\mathrm{Pb}$ : Presión de barreno, Mpa

$\sigma_{t d m r}$ : Resistencia a la tracción dinámica del macizo rocoso

$\emptyset_{b}$ : Diámetro del barreno, mm

$\emptyset_{e}$ : Diámetro del explosivo, mm

$\mathrm{K}_{\mathrm{A}}, \mathrm{K}_{\mathrm{SRF}}$ y $\mathrm{K}_{\mathrm{RMRb}}$ : Constantes para la sección del túnel, su profundidad y la influencia del RMR

\subsection{Valoración de las constantes $K_{A}, K_{S R F}$ y $K_{R M R b}$}

Inicialmente se indicó la influencia que debían tener la sección del túnel, su profundidad y su estado de fracturamiento, representado por el RMR, sobre la determinación del burden, el espaciamiento y la longitud de retaque.

$\mathrm{K}_{\mathrm{A}}$ es el indicador del efecto de la sección del túnel sobre los parámetros de diseño de la voladura; refleja las condiciones de confinamiento o relajamiento del material debido a su menor o mayor área expuesta, así como la posibilidad de interceptar un menor o mayor grado de anomalías presentes en el macizo; otra característica que también interviene es el efecto mismo de sus dimensiones y forma lo cual dificulta las operaciones de perforación. Este parámetro varía entre 1,0 (túneles de sección pequeña) y 1,2 (túneles de gran sección), Tabla 5.

$\mathrm{K}_{\mathrm{SRF}}$ es el indicador del efecto de la profundidad, el cual se encuentra directamente relacionado con el estado de confinamiento del macizo rocoso a las diferentes profundidades; en buena medida corresponde al Factor de Reducción de Esfuerzos de Barton cuando se relacionan la resistencia a la tracción de la roca intacta con respecto al esfuerzo vertical. Su rango varía desde 1,0 (túneles profundos) hasta 1,3 (túneles superficiales), Tabla $6 . \mathrm{K}_{\mathrm{RMRb}}$ corresponde al indicador por el estado del macizo rocoso conforme la clasificación de Bieniawski 1989 el cual a todas luces afecta los diseños de la voladura debido a su estado de fracturamiento. Este parámetro fluctúa entre 1,0 $(\mathrm{RMR}=\mathrm{I})$ y $1,10(\mathrm{RMR}=\mathrm{III})$, Tabla 7.

Tabla 5. Valoraciones del $\mathrm{K}_{\mathrm{A}}$.

\begin{tabular}{|l|c|c|}
\hline \multicolumn{1}{|c|}{ Descripción } & Sección del túnel, $\mathbf{m}^{2}$ & Valores de $\mathbf{K}_{\mathbf{A}}$ \\
\hline Túneles de muy pequeña sección & $<20$ & 1,00 \\
\hline Túneles de pequeña sección & $20-40$ & 1,05 \\
\hline Túneles de mediana sección & $40-60$ & 1,10 \\
\hline Túneles de gran sección & $60-80$ & 1,15 \\
\hline Túneles de muy grande sección & $>80$ & 1,20 \\
\hline
\end{tabular}

Tabla 6. Valoraciones del $\mathrm{K}_{\mathrm{SRF}}$.

\begin{tabular}{|l|c|c|c|}
\hline \multicolumn{4}{|c|}{ VALORES para KSRF } \\
\hline \multicolumn{1}{|c|}{ Descripción } & SRF & Prof. $\mathbf{m}$ & Valores de KSRF \\
\hline Cerca de la superficie & - & $0-300$ & 1,30 \\
\hline Niveles de esfuerzos bajos & 2,5 & $300-600$ & 1,20 \\
\hline Niveles de esfuerzos medios & 1,0 & $600-900$ & 1,10 \\
\hline Niveles de esfuerzos altos & 0,5 & $>900$ & 1,00 \\
\hline
\end{tabular}

Este comportamiento se deduce de innumerables escritos de este investigador (Profesor Bieniawski) y lo ratifica el profesor Carlos López Jimeno en su libro Manual de voladuras en túneles [8] el cual fue de obligada consulta en la presente investigación; de dicho texto extraemos la Tabla 8.

Tabla 7. Valoraciones del $\mathrm{K}_{\mathrm{RMRb}}$.

\begin{tabular}{|l|c|c|c|}
\hline \multicolumn{1}{|c|}{ Descripción } & \multicolumn{2}{c|}{ RMRb } & Valores de $\mathbf{K}_{\mathrm{RM} \text { IR }}$ \\
\hline Macizo rocoso muy bueno & $80-100$ & I & 1,00 \\
\hline Macizo rocoso bueno & $60-80$ & II & 1,05 \\
\hline Macizo rocoso regular & $40-60$ & III & 1,10 \\
\hline Macizo rocoso malo & $20-40$ & IV & N/A * \\
\hline Macizo rocoso muy malo & $0-20$ & V & N/A * \\
\hline
\end{tabular}

*N/A: No Aplica

Tabla 8. Número de barrenos versus sección del túnel y tipo de roca.

\begin{tabular}{|c|c|c|}
\hline \multirow{2}{*}{$\begin{array}{c}\text { Sección del túnel } \\
\mathbf{m}^{\mathbf{2}}\end{array}$} & \multicolumn{2}{|c|}{ Número de barrenos por pega } \\
\cline { 2 - 3 } & Roca Débil* & Roca Dura* \\
\hline 10 & $23-27$ & $35-50$ \\
\hline
\end{tabular}

* Roca Débil: roca blanda o muy fracturada. Roca Dura: roca muy resistente o masiva

Finalmente, con el burden definido, los coeficientes para el espaciamiento y el retaque se ilustran en la Tabla 9, así como la longitud de perforación recomendada.

Tabla 9. Coeficientes para el espaciamiento y el retaque en función del $\mathrm{RMRb}$.

\begin{tabular}{|l|c|c|c|}
\hline \multicolumn{1}{|c|}{ RMRb } & I & II & III \\
\hline$\beta$ (Constante de espaciamiento) & 0,90 & 0,95 & 1,00 \\
\hline (Constante de retaque) & 0,60 & 0,70 & 0,80 \\
\hline * Longitud promedia recomendada de Avance, $\mathbf{m}$ & $5-4$ & $4-3$ & $3-2$ \\
\hline
\end{tabular}


Estos coeficientes fueron definidos después de muchos intentos en los que confrontamos la sensibilidad de los resultados con tablas, gráficas y expresiones [10] que dan cuenta del número de barrenos según algunas variables como sección del túnel, tipo de roca o volabilidad. En conclusión, puede decirse que los valores extremos encontrados para estos coeficientes fueron:

a) Mayores valores: $1,1 \times 1,3 \times 1,2=1,72$ túnel de más de $80 \mathrm{~m}^{2}$ de sección, situado cerca de superficie (profundidad igual o menor de $300 \mathrm{~m}$ ) en un macizo rocoso RMR III.

b) Menores valores: $1,0 \times 1,0 \times 1,0=$ túnel de menos de $20 \mathrm{~m}^{2}$ de sección, situado a una profundidad mayor a $900 \mathrm{~m}$ en un macizo rocoso RMR I.

\subsection{Convalidación de resultados}

La convalidación de los resultados hallados según la expresión que venimos de proponer se fundamentó en confrontarlos con algunos datos referenciados en la bibliografía disponible y muy particularmente las gráficas que aparecen en la bibliografía [10], capítulo 4, en el que el autor presenta una serie de gráficas, a partir de las cuales diseñamos las Tablas 10 a 17, así como algunos patrones de voladura citados en la bibliografía después de analizar las características tenidas en cuenta para su diseño.

En principio, se puede afirmar que el número de barrenos depende tanto de la sección como del RMR (dificultad que ejerza el macizo rocoso para ser volado), así como del diámetro del barreno y su factor de acoplamiento, por ejemplo, para un túnel de $50 \mathrm{~m}^{2}$, barrenos de $45 \mathrm{~mm}$ y cargas acopladas, el número puede variar entre 60 y 90 según la competencia del macizo; esto es, aproximadamente un $30 \%$ de incremento. Una condición que podría aumentar el número de barrenos, está asociada al tamaño de bloque que pueda manejarse en el interior del túnel.

Tabla 10. Número de barrenos versus la volabilidad de la roca.

\begin{tabular}{|l|c|c|c|c|c|c|c|}
\hline \multicolumn{7}{|c|}{ Número de barrenos en función de la sección y } \\
la volabilidad de la roca para diámetros de perforación de 45mm. \\
\hline & \multicolumn{7}{|c|}{ Seción } \\
\hline & $<\mathbf{2 0 m}^{2}$ & $\mathbf{2 0 m}^{\mathbf{2}}$ & $\mathbf{4 0 m}^{2}$ & $\mathbf{6 0 m ^ { 2 }}$ & $\mathbf{8 0 m ^ { 2 }}$ & $\mathbf{1 0 0 m}^{2}$ & $>{\mathbf{1 0 0 m ^ { 2 }}}^{\mathbf{2}}$ \\
\hline Mala Volabilidad & 39 & 45 & 67 & 87 & 106 & 125 & 134 \\
\hline Buena Volabilidad & 45 & 52 & 79 & 102 & 125 & 148 & 160 \\
\hline
\end{tabular}

Tabla 11. Número de barrenos versus la sección y el diámetro de perforación

\begin{tabular}{|c|c|c|c|c|c|c|c|}
\hline \multicolumn{8}{|c|}{$\begin{array}{c}\text { Número de barrenos en función de } \\
\text { la sección y el diámetro de perforación. }\end{array}$} \\
\hline \multirow[b]{2}{*}{$\varphi(\mathbf{m m})$} & \multicolumn{7}{|c|}{ Sección } \\
\hline & $<20 \mathrm{~m}^{2}$ & $20 m^{2}$ & $40 m^{2}$ & $60 \mathrm{~m}^{2}$ & $80 \mathrm{~m}^{2}$ & $100 \mathrm{~m}^{2}$ & $>100 \mathrm{~m}^{2}$ \\
\hline 50 & 26 & 32 & 50 & 63 & 75 & 84 & 88 \\
\hline 40 & 30 & 37 & 58 & 73 & 87 & 97 & 102 \\
\hline 32 & 35 & 43 & 67 & 85 & 100 & 112 & 117 \\
\hline
\end{tabular}

Tabla 12. Número de barrenos versus la sección y el tipo de roca.

\begin{tabular}{|c|c|c|c|c|c|}
\hline \multicolumn{6}{|c|}{ Número de barrenos en función de la sección y el tipo de roca. } \\
\hline & \multicolumn{5}{|c|}{ Sección } \\
\hline & $<20 \mathrm{~m}^{2}$ & $\mathbf{2 0} \mathbf{m}^{2}$ & $40 \mathrm{~m}^{2}$ & $60 \mathrm{~m}^{2}$ & $80 \mathrm{~m}^{2}$ \\
\hline Mica Esquistos & 40 & 58 & 72 & 78 & 80 \\
\hline Granitos & 35 & 52 & 66 & 70 & 73 \\
\hline Areniscas & 31 & 47 & 60 & 65 & 66 \\
\hline Pizarra & 29 & 42 & 55 & 60 & 61 \\
\hline
\end{tabular}

Tabla 13. Perforación específica versus la sección y la volabilidad para barrenos de 45 y $64 \mathrm{~mm}$.

\begin{tabular}{|c|c|c|c|c|c|c|c|}
\hline \multicolumn{8}{|c|}{$\begin{array}{l}\text { Perforación especifica }\left(\mathrm{ml} / \mathrm{m}^{3}\right) \text { para barrenos de } 45 \text { y } 64 \mathrm{~mm} \\
\text { en función de la sección y la volabilidad. }\end{array}$} \\
\hline & \multicolumn{7}{|c|}{ Sección } \\
\hline & $<20 \mathrm{~m}^{2}$ & $20 \mathrm{~m}^{2}$ & $40 \mathrm{~m}^{2}$ & $60 \mathrm{~m}^{2}$ & $80 \mathrm{~m}^{2}$ & $100 \mathrm{~m}^{2}$ & $>100 \mathrm{~m}^{2}$ \\
\hline Mala Volabilidad, $\varphi=64 \mathrm{~mm}$ & 6,90 & 6,00 & 4,10 & 3,65 & 3,40 & 3,20 & 3,20 \\
\hline Buena Volabilidad, $\varphi=64 \mathrm{~mm}$ & 5,85 & 5,00 & 3,50 & 3,10 & 2,90 & 2,75 & 2,70 \\
\hline Mala Volabilidad, $\varphi=45 \mathrm{~mm}$ & 4,90 & 4,25 & 2,95 & 2,55 & 2,40 & 2,35 & 2,30 \\
\hline Buena Volabilidad, $\varphi=45 \mathrm{~mm}$ & 4,20 & 3,70 & 2,45 & 2,15 & 2,05 & 2,00 & 2,00 \\
\hline
\end{tabular}

Tabla 14. Consumo específico versus la sección para barrenos de 30, 40 y $50 \mathrm{~mm}$.

\begin{tabular}{|c|c|c|c|c|c|c|c|}
\hline \multicolumn{8}{|c|}{$\begin{array}{l}\text { Consumo específico }\left(\mathrm{Kg} / \mathrm{m}^{3}\right) \text { de explosivo } \\
\text { en función de la sección y el diámetro de perforación }\end{array}$} \\
\hline & \multicolumn{7}{|c|}{ Sección } \\
\hline & $<20 \mathrm{~m}^{2}$ & $20 \mathbf{m}^{2}$ & $40 m^{2}$ & $60 \mathrm{~m}^{2}$ & $80 m^{2}$ & $100 \mathrm{~m}^{2}$ & $>100 \mathrm{~m}^{2}$ \\
\hline$\Phi=50 \mathrm{~mm}$ & 3,00 & 2,10 & 1,45 & 1,30 & 1,25 & 1,15 & 1,10 \\
\hline$\Phi=40 \mathrm{~mm}$ & 2,80 & 1,90 & 1,30 & 1,20 & 1,10 & 1,05 & 1,00 \\
\hline$\Phi=30 \mathrm{~mm}$ & 2,60 & 1,60 & 1,10 & 1,05 & 1,00 & 0,90 & 0,90 \\
\hline
\end{tabular}

Tabla 15. Consumo específico versus la sección y el tipo de roca.

\begin{tabular}{|c|c|c|c|c|c|}
\hline Consumo específico $\left(\mathbf{K g} / \mathbf{m}^{\mathbf{3}}\right)$ de explosivo en función de la sección y el tipo de roca \\
\hline Sección & $\mathbf{4 m}^{\mathbf{2}}$ & $\mathbf{1 2 \mathbf { m } ^ { \mathbf { 2 } }}$ & $\mathbf{2 0 \mathbf { m } ^ { \mathbf { 2 } }}$ & $\mathbf{2 8 \mathbf { m } ^ { \mathbf { 2 } }}$ & $\mathbf{3 6 \mathbf { m } ^ { \mathbf { 2 } }}$ \\
\hline \multicolumn{7}{|c|}{ Consumo Específico $\left(\mathrm{Kg} / \mathbf{m}^{\mathbf{3}}\right)$} \\
\hline Mica Esquistos & 5,35 & 3,50 & 2,30 & 1,80 & 1,65 \\
\hline Granitos & 4,40 & 2,80 & 1,85 & 1,50 & 1,35 \\
\hline Areniscas & 3,80 & 2,25 & 1,55 & 1,25 & 1,20 \\
\hline Pizarra & 3,20 & 1,85 & 1,3 & 1,00 & 0,90 \\
\hline
\end{tabular}

Tabla 16. Consumo específico versus la sección y volabilidad para barrenos de $45 \mathrm{~mm}$

\begin{tabular}{|c|c|c|c|c|c|c|c|}
\hline \multicolumn{7}{|c|}{$\begin{array}{c}\text { Consumo específico }\left(\mathrm{Kg} / \mathbf{m}^{\mathbf{3}}\right) \text { de explosivos encartuchados }(\boldsymbol{\varphi}=\mathbf{4 5 m m}) \\
\text { en función de la sección y la volabilidad de la roca }\end{array}$} \\
\hline & \multicolumn{7}{|c|}{ Sección } \\
\hline & $<\mathbf{2 0 \mathbf { m } ^ { \mathbf { 2 } }}$ & $\mathbf{2 0 \mathbf { m } ^ { \mathbf { 2 } }}$ & $\mathbf{4 0 \mathbf { m } ^ { 2 }}$ & $\mathbf{6 0 \mathbf { m } ^ { 2 }}$ & $\mathbf{8 0 \mathbf { m } ^ { 2 }}$ & $\mathbf{1 0 0 \mathbf { m } ^ { \mathbf { 2 } }}$ & $>1 \mathbf{0 0 \mathbf { m } ^ { \mathbf { 2 } }}$ \\
\hline Mala Volabilidad & 2,70 & 2,00 & 1,50 & 1,35 & 1,20 & 1,15 & 1,10 \\
\hline Buena Volabilidad & 2,40 & 1,80 & 1,40 & 1,20 & 1,10 & 1,00 & 1,00 \\
\hline
\end{tabular}


Tabla 17. Consumo específico versus la sección y la volabilidad

\begin{tabular}{|c|c|c|c|c|c|c|c|}
\hline \multicolumn{7}{|c|}{$\begin{array}{c}\text { Consumo especifico }\left(\mathbf{K g} / \mathbf{m}^{3}\right) \text { de } \mathbf{A N F O} \\
\text { en función de la sección y la volabilidad de la roca }\end{array}$} \\
\hline & \multicolumn{7}{|c|}{ Sección } \\
\hline & $<\mathbf{2 0 m}^{2}$ & $\mathbf{2 0 m}^{2}$ & $\mathbf{4 0 m}^{2}$ & $\mathbf{6 0 m ^ { 2 }}$ & $\mathbf{8 0 m ^ { 2 }}$ & $\mathbf{1 0 0 m}^{2}$ & $>1 \mathbf{1 0 0 m}^{2}$ \\
\hline Mala Volabilidad & 2,55 & 2,30 & 1,65 & 1,75 & 1,30 & 1,25 & 1,20 \\
\hline Buena Volabilidad & 2,25 & 2,00 & 1,40 & 1,35 & 1,10 & 1,05 & 1,00 \\
\hline
\end{tabular}

\subsection{Patrones de voladura}

Como es apenas normal, en la bibliografía no es muy común encontrar todos los parámetros que estamos proponiendo para alimentar nuestra expresión y muy particularmente por la dificultad en la determinación de la resistencia a la tracción dinámica del macizo rocoso; no obstante, en algunos casos se puede tener una información suficiente que puede utilizarse con fines de ajustar los parámetros de la expresión propuesta. Para el caso colombiano la dificultad es mayor debido al secreto de estado que envuelve estos diseños.

En la serie de Figuras 1 y 2 y Tablas 18 y 19 se quieren ilustrar las variaciones que sufren los patrones de voladura según los diversos parámetros en los que se soporta la expresión propuesta. Obsérvense el número y distribución de barrenos así como el valor del burden y el del espaciamiento.

Tabla 18. TÚNEL DE PEQUEÑA SECCIÓN $\left(19.60 \mathrm{~m}^{2}, \mathrm{~K}_{\mathrm{A}}=1.0\right)$ Diámetro de perforación: $45 \mathrm{~mm}$

\begin{tabular}{|l|c|c|c|c|c|c|}
\hline & \multicolumn{3}{|c|}{ INDUGEL PLUS AP } & \multicolumn{3}{c|}{ ANFO } \\
\hline & RMR I & RMR II & RMR III & RMR I & RMR II & RMR III \\
\hline Burden, m & 0.72 & 0.96 & 1.35 & 0.47 & 0.63 & 0.88 \\
\hline Espaciamiento, $\mathbf{m}$ & 0.65 & 0.91 & 1.35 & 0.42 & 0.60 & 0.88 \\
\hline Longitud de perforación, $\mathbf{m}$ & 3.00 & 2.00 & 1.50 & 3.00 & 2.00 & 1.50 \\
\hline Número de barrenos & 53 & 43 & 36 & 83 & 67 & 47 \\
\hline
\end{tabular}

Tabla 19. TÚNEL DE GRAN SECCIÓN $\left(82.23 \mathrm{~m}^{2}, \mathrm{~K}_{\mathrm{A}}=1.2\right)$. Diámetro de perforación: $45 \mathrm{~mm}$

\begin{tabular}{|l|c|c|c|c|c|c|}
\hline & \multicolumn{2}{|c|}{ INDUGEL PLUS AP } & \multicolumn{3}{c|}{ ANFO } \\
\hline & RMR I & RMR II & RMR III & RMR I & RMR II & RMR III \\
\hline Burden, $\mathbf{m}$ & $\mathbf{0 . 8 6}$ & $\mathbf{1 . 1 5}$ & $\mathbf{1 . 6 2}$ & $\mathbf{0 . 5 6}$ & $\mathbf{0 . 7 5}$ & $\mathbf{1 . 0 5}$ \\
\hline Espaciamiento, $\mathbf{m}$ & $\mathbf{0 . 7 7}$ & $\mathbf{1 . 0 9}$ & $\mathbf{1 . 6 2}$ & $\mathbf{0 . 5 0}$ & $\mathbf{0 . 7 1}$ & $\mathbf{1 . 0 5}$ \\
\hline Longitud de perforación, $\mathrm{m}$ & $\mathbf{4 . 5 0}$ & $\mathbf{3 . 5 0}$ & $\mathbf{2 . 5 0}$ & $\mathbf{4 . 5 0}$ & 3.50 & $\mathbf{2 . 5 0}$ \\
\hline Número de barrenos & 154 & 101 & $\mathbf{7 2}$ & $\mathbf{3 1 8}$ & $\mathbf{1 8 3}$ & $\mathbf{1 0 4}$ \\
\hline
\end{tabular}

\section{Conclusiones y recomendaciones}

Para hacer uso de la expresión propuesta se necesita una buena caracterización tanto de la roca intacta como del macizo rocoso y muy particularmente sus comportamientos dinámicos a la tracción. Esta expresión demanda igualmente del conocimiento de las propiedades del barreno y muy particularmente su llenado, pues más desacoplada es la carga, más pérdida se tendrá de la energía del explosivo.

La expresión también hace intervenir unas constantes dependientes del nivel de confinamiento al que está sometido el túnel, así como su sección y, por supuesto, su estado de fracturamiento reflejado en el RMR.
No se recomienda el uso de explosivos para macizos rocosos tipo IV y $\mathrm{V}$ en la clasificación del RMR. Esta expresión puede utilizarse para racionalizar los costos y tiempos, así como la estabilidad y buen contorno de la sección del túnel.

En el desarrollo de la expresión no se tuvieron en cuenta los barrenos adicionales del contorno del túnel, al utilizar el precorte (el cual estamos recomendando con vehemencia), para no alterar el número de barrenos que aparece en la literatura y que sirvieron para comparación.

Si bien es cierto los análisis realizados en el presente documento se llevaron a cabo para una voladura tradicional, con el fin de confrontar el número de barrenos con secciones y tablas disponibles en la literatura técnica, recomendamos que siempre se lleve a cabo el precorte, pues es el único que puede garantizar un buen perfil, el cual estaría aumentando el número de barrenos en aproximadamente unos 15, dependiendo del RMR y de la sección; esto es, los barrenos del perfil se estarían multiplicando por 2, aproximadamente, Figura 3.

Recomendamos que esta expresión se ponga a prueba en nuestros macizos rocosos colombianos, con la finalidad de ajustar los parámetros, si fuere necesario en procura de voladuras más económicas, limpias y eficientes.

\section{Referencias}

[1] Correa Á., Rueda J.F., Rodríguez F.B.; En: "Manual para el diseño, construcción, operación y mantenimiento de túneles de carretera para Colombia, INVIAS, Edición (2021), Capítulo 6, 6.7.2 Esquema de voladura, Pagina 220.

[2] López J.C. (1994) "Manual de perforación y voladura de rocas". Instituto Tecnológico GeoMinero de España

[3] Borquez G.V. (1981), "Estimation of drilling and blasting cost - An analysis and prediction model", Engineering and Mining Journal, January 1981, pp83-89.

[4] López J.C., (2013) "Manual de perforación en túneles". E.T.S.I. Minas Universidad Politécnica de Madrid, España.

[5] Diéguez G.Y., et al., "Campo tenso-deformacional para voladuras con cordón detonante en el laboreo de túneles", Revista Minería y Geología, Vol. 29 No.3 (2013), p. 38-55.

[6] Hoek E., (2000). "Rock Mass Properties". En Practical Rock Engineering

[7] González de Vallejo, L,I. (2006). "Ingeniería Geológica", Pearson Educación, Madrid

[8] INDUMIL. (2009). "Guía del Explosivista"

[9] INDUMIL. (2018). "Manual para explosivos Industriales"

[10] López J.C, (2010) “Manual de voladuras en túneles”. E.T.S.I. Minas Universidad Politécnica de Madrid, España 


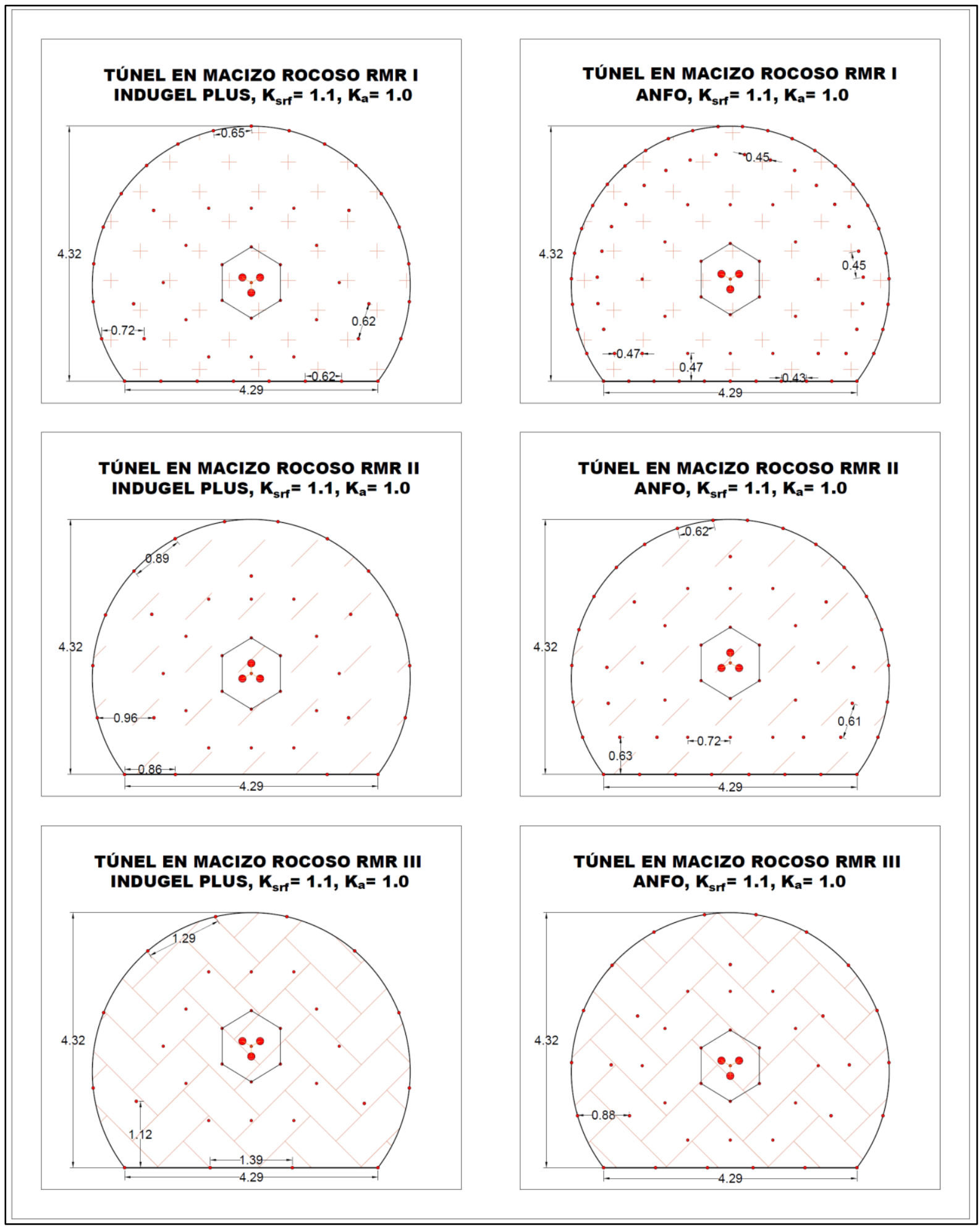

Figura 1. Esquemas de perforación y voladura para diferentes tipos de RMR, y sección de $19.60 \mathrm{~m}^{2}$. Fuente: Autor. 

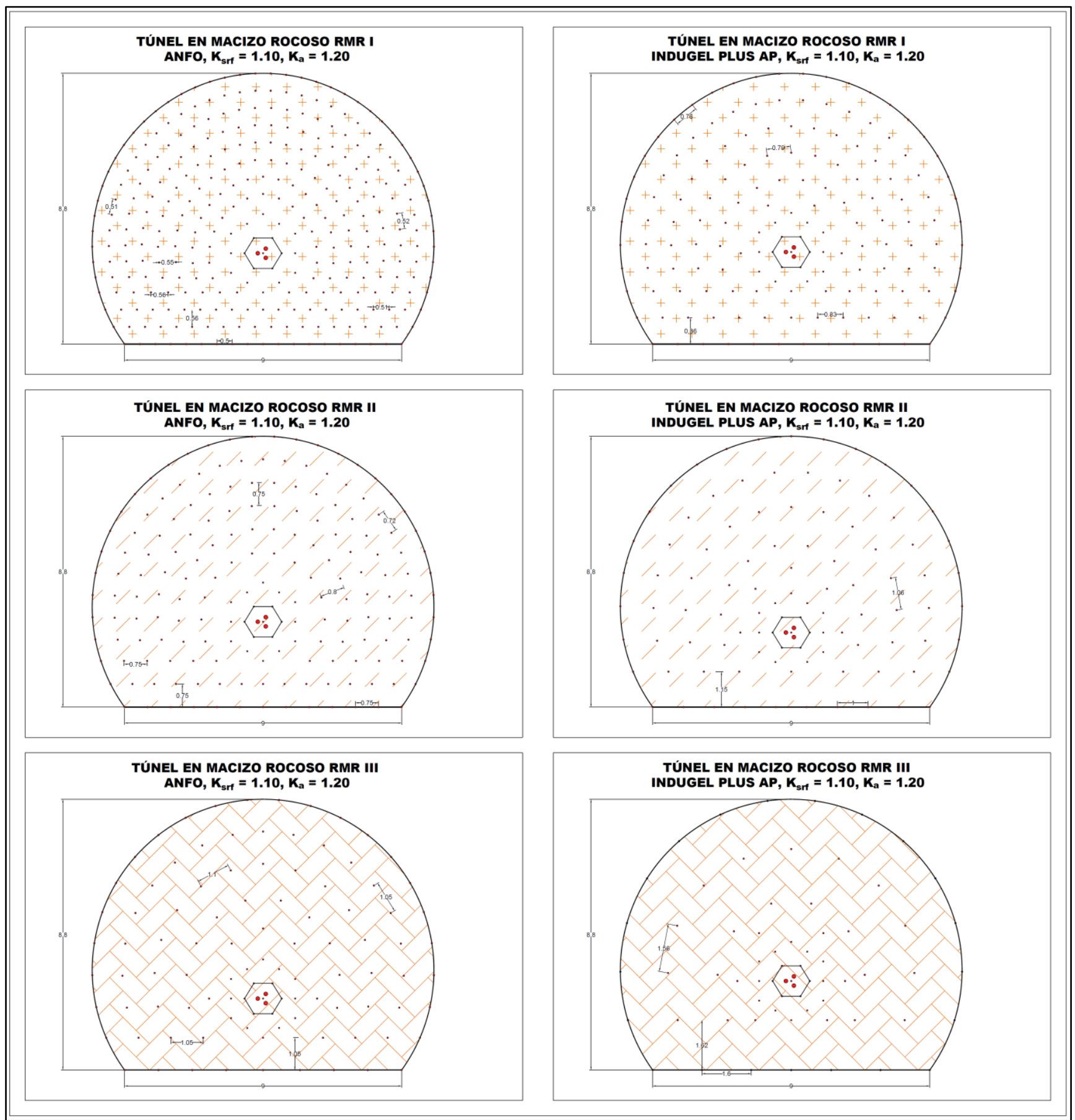

Figura 2. Esquemas de perforación y voladura para diferentes tipos de RMR, y sección de $82.23 \mathrm{~m}^{2}$. Fuente: Autor. 


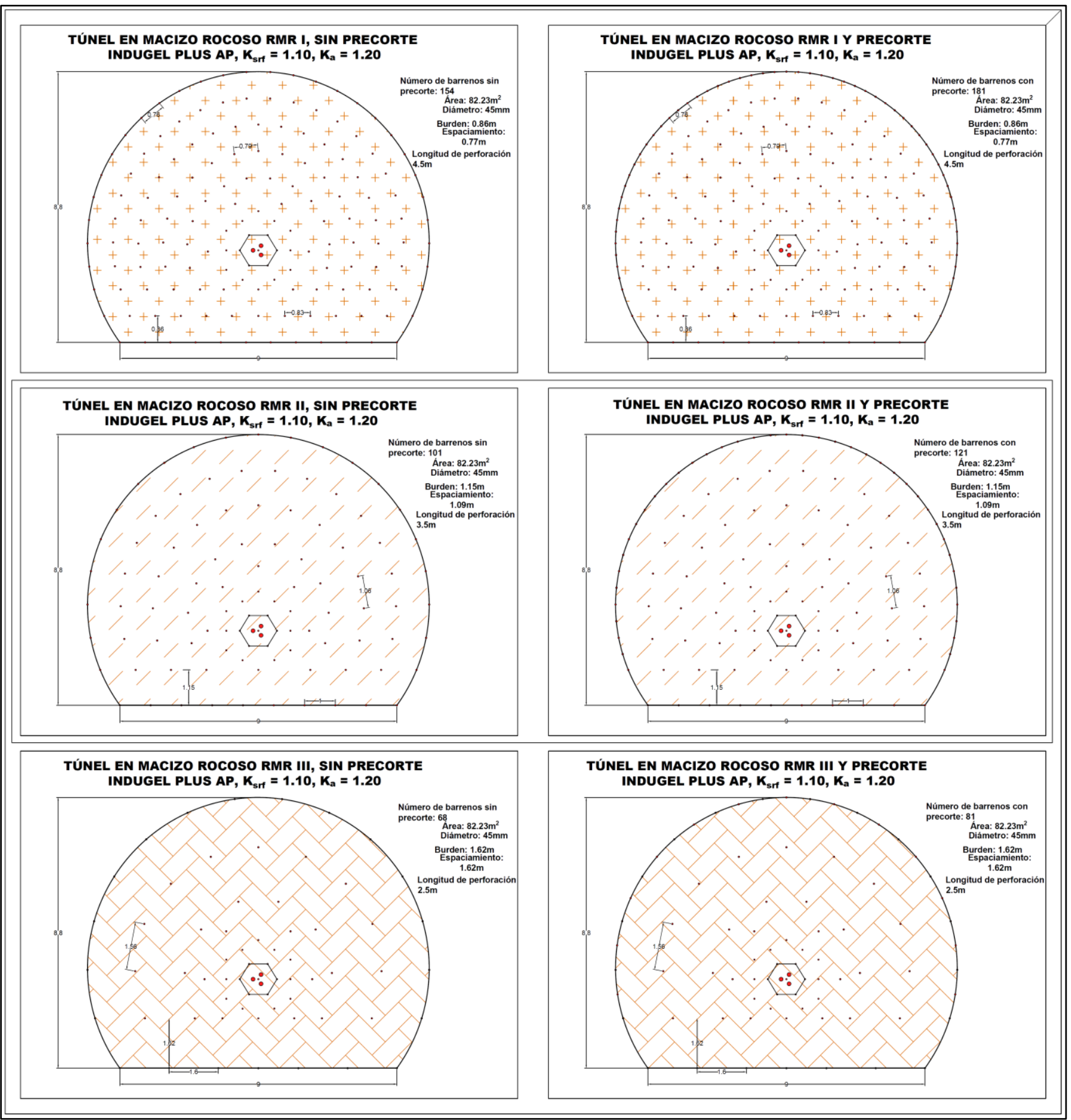

Figura 3. Esquemas de perforación y voladura para diferentes tipos de RMR, y sección de $82.23 \mathrm{~m}^{2}$, sin precorte y con precorte. Fuente: Autor. 